\title{
Some Results of Real Symmetric Semi-Definite Matrix Traces
}

\author{
Feng Zhang*, Jinli Xu
}

Department of mathematics, Northeast Forestry University, Harbin, China, 150040

*Corresponding Author: Feng Zhang, Department of mathematics, Northeast Forestry University, Harbin, China, 150040

Abstract: The first part of this paper is to explore the inequalities of the traces of real symmetric semi-positive definite matrices under partial order relations. We find more general conclusions from special results

Keywords: Semi-definite matrix Trace inequality Partial ordering

\section{MAIn Results}

In the partial order relationship, we calculate the product of the semi-positive definite matrices with smaller powers, and compare the size relationship.

If $\mathrm{A}$ and $\mathrm{B}$ are real symmetric semi-positive definite matrices (this part is discussed under the condition of real symmetric semi-positive definite matrices, and we will not

Emphasize it later), $\mathrm{A} \geq \mathrm{B}, \quad$ then $\operatorname{tr}\left(\mathrm{A}^{2}-\mathrm{AB}\right)=\operatorname{tr} \mathrm{A}(\mathrm{A}-\mathrm{B}) \geq 0, \quad \operatorname{tr}\left(\mathrm{AB}-\mathrm{B}^{2}\right)=\operatorname{tr}(\mathrm{A}-\mathrm{B}) \mathrm{B} \geq 0$.

So, we get

$\operatorname{tr} A^{2} \geq \operatorname{tr} A B \geq \operatorname{tr} B^{2}$. Next, we see that

$\operatorname{tr}\left(\mathrm{A}^{2} \mathrm{~B}-\mathrm{AB}^{2}\right)=\operatorname{tr}[\mathrm{AB}(\mathrm{A}-\mathrm{B})]=\operatorname{tr} \mathrm{B}(\mathrm{A}-\mathrm{B}) \mathrm{A}=\operatorname{tr} \mathrm{B}(\mathrm{A}-\mathrm{B}) \mathrm{B}+\operatorname{tr} \mathrm{B}(\mathrm{A}-\mathrm{B})(\mathrm{A}-\mathrm{B}) \geq 0$,

Then we prove $\operatorname{tr} \mathrm{A}^{2} \mathrm{~B} \geq \operatorname{tr} \mathrm{AB}^{2}$.

And it is not difficult to calculate that

$\operatorname{tr} \mathrm{A}^{3}-\operatorname{tr} \mathrm{A}^{2} \mathrm{~B}=\operatorname{tr} \mathrm{A}^{2}(\mathrm{~A}-\mathrm{B}) \geq 0, \quad \operatorname{tr} \mathrm{AB}^{2}-\operatorname{tr} \mathrm{B}^{3}=\operatorname{tr}(\mathrm{A}-\mathrm{B}) \mathrm{B}^{2} \geq 0$.

So we can get a sequence relationship that $\operatorname{tr} A^{3} \geq \operatorname{tr} A^{2} B \geq \operatorname{tr} A B^{2} \geq \operatorname{tr} B^{3}$.

$$
\begin{aligned}
& \operatorname{tr}\left(A^{3} B-A B^{3}\right)=\operatorname{tr}\left(A^{2} B-A B^{2}\right)(A+B)=\operatorname{tr}(A-B) B(A+B) A \\
& =\operatorname{tr}(A-B) B(A+B) B+\operatorname{tr}(A-B) B(A+B)(A-B) \\
& =\operatorname{tr}(A-B) B(A+B) B+\operatorname{tr} B(A+B)(A-B)^{2}=\operatorname{tr}(A-B) B(A+B) B+\operatorname{tr} B^{2}(A-B)^{2}+\operatorname{tr} B A(A-B)^{2} \\
& =\operatorname{tr}(A-B) B(A+B) B+\operatorname{tr} B^{2}(A-B)^{2}+\operatorname{tr} A(A-B)^{2} B \\
& =\operatorname{tr}(A-B) B(A+B) B+\operatorname{tr} B^{2}(A-B)^{2}+\operatorname{tr} B(A-B)^{2} B+\operatorname{tr}(A-B)^{3} B \geq 0
\end{aligned}
$$

So $\operatorname{tr} A^{3} B \geq \operatorname{tr} A B^{3}$

$\operatorname{tr}\left(A^{2} B^{2}-A B^{3}\right)=\operatorname{tr} B A(A-B) B=\operatorname{tr} B^{3}(A-B)+\operatorname{tr} B^{2}(A-B)^{2}$.

According to $\mathrm{B}^{3}(\mathrm{~A}-\mathrm{B}), \mathrm{B}^{2}(\mathrm{~A}-\mathrm{B})^{2}$ are similar to the non-negative diagonal matrix, 
so $\operatorname{tr} B^{3}(A-B)+\operatorname{tr} B^{2}(A-B)^{2} \geq 0$, 于是可以得到 $\operatorname{tr}\left(A^{2} B^{2}-A B^{3}\right) \geq 0$

$\operatorname{tr}\left(A^{3} B-A^{2} B^{2}\right)=\operatorname{tr} A(A-B) B A$

$$
=\operatorname{tr} B(A-B) B B+\operatorname{tr} B(A-B) B(A-B)+\operatorname{tr}(A-B)^{2} B A
$$

We can get $\operatorname{tr} B(A-B) B B \geq 0, \operatorname{tr} B(A-B) B(A-B) \geq 0$ by $A \geq B$.

And $\operatorname{tr}(A-B)^{2} B A=\operatorname{tr} A(A-B)^{2} B=\operatorname{tr} B(A-B)^{2} B+\operatorname{tr}(A-B)^{3} B \geq 0$, so we say $\operatorname{tr}\left(A^{3} B-A^{2} B^{2}\right) \geq 0$.

So we can get that $\operatorname{tr} A^{3} B \geq \operatorname{tr} A^{2} B^{2} \geq \operatorname{tr} A B^{3}$, we use the same methods can prove $\operatorname{tr} A^{4} \geq \operatorname{tr} A^{3} B \geq \operatorname{tr} A^{2} B^{2} \geq \operatorname{tr} A B^{3} \geq \operatorname{tr} B^{4}$

$\operatorname{tr}\left(A^{4} B-A B^{4}\right)=\operatorname{tr}\left(A^{2} B-A B^{2}\right)\left(A^{2}+B^{2}\right)+\operatorname{tr}\left(A^{3} B^{2}-A^{2} B^{3}\right)$

$\operatorname{tr}\left(A^{2} B-A B^{2}\right)\left(A^{2}+B^{2}\right)=\operatorname{tr}(A-B) B\left(A^{2}+B^{2}\right) A$

$$
=\operatorname{tr}(A-B) B\left(A^{2}+B^{2}\right) B+\operatorname{tr} B A^{2}(A-B)^{2}+\operatorname{tr} B^{3}(A-B)^{2}
$$

$\operatorname{tr}\left(A^{3} B^{2}-A^{2} B^{3}\right)=\operatorname{tr} B^{2}(A-B) A^{2}$,

So $\operatorname{tr}\left(A^{4} B-A^{3} B^{2}\right) \geq 0$,

For $\operatorname{tr}\left(A^{4} B-A^{3} B^{2}\right)=\operatorname{tr} A^{3}(A-B) B=\operatorname{tr} A B(A-B) B A+\operatorname{tr} A(A-B)^{2} B A$

And $\operatorname{tr} A(A-B)^{2} B A=\operatorname{tr} B(A-B)^{2} B A+\operatorname{tr}(A-B)^{3} B A$

$$
=\operatorname{tr} B(A-B)^{2} B A+\operatorname{tr} B(A-B)^{3} B+\operatorname{tr}(A-B)^{4} B \geq 0
$$

Then $\operatorname{tr}\left(A^{4} B-A^{3} B^{2}\right)=\operatorname{tr} A^{3}(A-B) B=\operatorname{tr} A B(A-B) B A+\operatorname{tr} A(A-B)^{2} B A \geq 0$

Finally, we know that $\operatorname{tr} B A^{2}(A-B)^{2}+\operatorname{tr} B^{2}(A-B) A^{2}=\operatorname{tr}\left(A^{4} B-A^{3} B^{2}\right) \geq 0$

Taking these circumstances, we get that $\operatorname{tr}\left(A^{4} B-A B^{4}\right) \geq 0$.

Some of the above formulas give us inspiration:

If $\mathrm{s}+\mathrm{t}=\mathrm{k}, \mathrm{A}, \mathrm{B}$ are real symmetric positive definite matrices (guaranteed inequality meaningful) $\mathrm{A}$ $\geq B$, Is there $\operatorname{tr} A^{k} \geq \operatorname{tr} A^{k-1} B \geq \operatorname{tr} A^{k-2} B^{2} \geq \ldots . \geq \operatorname{tr} A B^{k-1} \geq \operatorname{tr} B^{k}$ established?

Let us give a positive answer to this question.

Theorem If $\mathrm{s}+\mathrm{t}=\mathrm{k}, \mathrm{s}, \mathrm{t} \in \mathrm{N}, \mathrm{A}, \mathrm{B}$ are real symmetric positive definite matrices, $\mathrm{A} \geq \mathrm{B}$, 
Then $\operatorname{tr} A^{k} \geq \operatorname{tr} A^{k-1} B \geq \operatorname{tr} A^{k-2} B^{2} \geq \ldots \geq \operatorname{tr} A B^{k-1} \geq \operatorname{tr} B^{k}$ is established.

Let $m$ be an integer greater than $1, A, B$ are real symmetric positive definite matrices, then $\operatorname{tr}\left(A^{m} B\right) \geq \operatorname{tr}\left(A^{m-1} B^{2}\right)$

$\operatorname{tr}\left(A^{m} B\right)-\operatorname{tr}\left(A^{m-1} B^{2}\right)=\operatorname{tr}\left[A^{m-1}(A-B) B\right]=\operatorname{tr}\left[A^{m-2} B(A-B) B\right]+\operatorname{tr}\left[A^{m-2}(A-B)^{2} B\right]$ $\operatorname{tr}\left[A^{m-2} B(A-B) B\right] \geq 0$, If $\operatorname{tr}\left[A^{m-2}(A-B)^{2} B\right] \geq 0$, Then the proposition can be proved. $\operatorname{tr}\left[A^{m-2}(A-B)^{2} B\right]=\operatorname{tr}\left[A^{m-3} B(A-B)^{2} B\right]+\operatorname{tr}\left[A^{m-3}(A-B)^{3} B\right]$, From the equation, we realize that we should prove that $\operatorname{tr}\left[A^{m-3}(A-B)^{3} B\right] \geq 0$, Decompose for $\operatorname{tr}\left[A^{m-3}(A-B)^{3} B\right]$, And summarize it, finally we only prove $\operatorname{tr}\left[A(A-B)^{m-1} B\right] \geq 0$,

and $\operatorname{tr}\left[A(A-B)^{m-1} B\right]=\operatorname{tr}\left[B(A-B)^{m-1} B\right]+\operatorname{tr}\left[(A-B)^{m} B\right] \geq 0$.

So we get that $\operatorname{tr}\left(A^{m} B\right) \geq \operatorname{tr}\left(A^{m-1} B^{2}\right)$.

$\operatorname{tr}\left(A^{m-1} B^{2}\right)-\operatorname{tr}\left(A^{m-2} B^{3}\right)=\operatorname{tr}\left[A^{m-2}(A-B) B^{2}\right]$,

$\operatorname{tr}\left[A^{m-2}(A-B) B^{2}\right]=\operatorname{tr}(A-B) A^{m-3}(A-B) B^{2}+\operatorname{tr} B A^{m-3}(A-B) B^{2}$

$\operatorname{tr}(A-B) A^{m-3}(A-B) B^{2} \geq 0$ can be obtained from the properties of semi-definite matrix traces.

Next, $\operatorname{tr} B A^{m-3}(A-B) B^{2}=\operatorname{tr} A^{m-3}(A-B) B^{3}=\operatorname{tr}(A-B) A^{m-4}(A-B) B^{2}+\operatorname{tr} B A^{m-4}(A-B) B^{2}$

We repeat the process above, and we can see $\operatorname{tr}\left(A^{m-1} B^{2}\right) \geq \operatorname{tr}\left(A^{m-2} B^{3}\right)$ by $\operatorname{tr} B(A-B) B^{2} \geq 0$

Use the same way, we also can prove $\operatorname{tr}\left(A^{m-2} B^{3}\right) \geq \operatorname{tr}\left(A^{m-3} B^{4}\right) \ldots$

We assume that $s \geq 1$ is a positive integer, then $\operatorname{tr}\left(A^{s+1} B^{s}\right) \geq \operatorname{tr}\left(A^{s} B^{s+1}\right)$

For $\operatorname{tr}\left(A^{s+1} B^{s}\right)-\operatorname{tr}\left(A^{s} B^{s+1}\right)=\operatorname{tr} A^{s}(A-B) B^{s}$

$$
=\operatorname{tr}(A-B) A^{s-1}(A-B) B^{s}+\operatorname{tr} B A^{s-1}(A-B) B^{s}
$$




$$
\begin{aligned}
& =\operatorname{tr}(A-B) A^{s-1}(A-B) B^{s}+\operatorname{tr}(A-B) A^{s-2}(A-B) B^{s+1}+\operatorname{tr} B A^{s-2}(A-B) B^{s+1} \\
& \operatorname{tr} B A^{s-2}(A-B) B^{s+1}=\operatorname{tr}(A-B) A^{s-3}(A-B) B^{s+2}+\operatorname{tr} B A^{s-3}(A-B) B^{s+2}
\end{aligned}
$$

We repeat this process over and over again,

then we can see $\operatorname{tr} B A(A-B) B^{2 s-1}=\operatorname{tr}(A-B)(A-B) B^{2 s}+\operatorname{tr} B(A-B) B^{2 s}$, and

$$
\operatorname{tr}(A-B)(A-B) B^{2 s}=\operatorname{tr}(A-B) B^{2 s}(A-B) \geq 0, \operatorname{tr} B(A-B) B^{2 s}=\operatorname{tr}(A-B) B^{2 s+1} \geq 0
$$

Then we prove that $\operatorname{tr}\left(A^{s+1} B^{s}\right) \geq \operatorname{tr}\left(A^{s} B^{s+1}\right)$.

So we can prove that $\operatorname{tr} A^{k} \geq \operatorname{tr} A^{k-1} B \geq \operatorname{tr} A^{\dot{k}-2} B^{2} \geq \ldots . \operatorname{tr} A B^{k-1} \geq \operatorname{tr} B^{k}$.

\section{REFERENCES}

[1] Pritchina E A, Gritsan N P, Bally T. Matrix isolation and computational study of the photochemistry of pazidoaniline.[J]. Physical Chemistry Chemical Physics Pccp, 2006, 8(6):719-727.

[2] Micchelli C A. Interpolation of scattered data: Distance matrices and conditionally positive definite functions[J]. Constructive Approximation, 1986, 2(1):11-22.

[3] Wendland H. Piecewise polynomial, positive definite and compactly supported radial functions of minimal degree[J]. Advances in Computational Mathematics, 1995,4(1):389-396.

[4] Bai Z Z, Golub G H, Li C K. Convergence Properties of Preconditioned Hermitian andSkewHermitian Splitting Methods for Non-Hermitian Positive Semidefinite Matrices[J]. Mathematics of Computation, 2007, 76(257):287-298.

[5] ARSIGNY, Vincent, FILLARD, et al. GEOMETRIC MEANS IN A NOVEL VECTOR SPACE STRUCTURE ON SYMMETRIC POSITIVE-DEFINITE MATRICES[J]. Siam Journal on Matrix Analysis \& Applications, 2011, 29(1):328-347.

[6] Belmega E V, Lasaulce S, Debbah M. A trace inequality for positive definite matrices[J]. Journal of Inequalities in Pure \& Applied Mathematics, 2010, 10(1).

[7] Bîrsan M. Sum of squared logarithms - an inequality relating positive definite matrices and their matrix logarithm[J]. Journal of Inequalities \& Applications, 2013, 2013(1):1-16.

Citation: Feng Zhang \& Jinli Xu (2019). Some Results of Real Symmetric Semi-Definite Matrix Traces. International Journal of Scientific and Innovative Mathematical Research (IJSIMR), 7(5), pp.10-13. http://dx.doi.org/ 10.20431/2347-3142.0705003

Copyright: () 2019 Authors, this is an open-access article distributed under the terms of the Creative Commons Attribution License, which permits unrestricted use, distribution, and reproduction in any medium, provided the original author and source are credited. 